\title{
RHPN2 Promotes Malignant Cell Behaviours in Ovarian Cancer by Activating STAT3 Signalling
}

This article was published in the following Dove Press journal: OncoTargets and Therapy

\author{
Fengsheng $\mathrm{Yu}^{\prime}$ \\ Pingping Qiao ${ }^{2}$ \\ Guangjie Yin' \\ Yewu Sun' \\ Xiao Yu' \\ Xin Sun' \\ Yijing Chu (D' \\ Yankui Wang (D)
}

'Department of Gynaecology, The Affiliated Hospital of Qingdao University, Qingdao, People's Republic of China; ${ }^{2}$ Department of Neurology, The Third People's Hospital of Qingdao, Qingdao, People's Republic of China
Correspondence: Yankui Wang; Yijing

Chu

Department of Gynaecology, The

Affiliated Hospital of Qingdao University,

16 Jiangsu Road, Qingdao 26606I,

People's Republic of China

Tel +86-I866I808529; +86-I 7853297008

Email ykwqd@hotmail.com;

chuyijing@।63.com
Background: Ovarian cancer is the fifth most common cause of cancer-related deaths and accounts for $3 \%$ of cancer cases occurring in women. Therefore, determining the underlying genes that can promote ovarian cancer progression is of great urgency. It has been reported that RHPN2 promotes tumour progression in various types of cancer, but its role in ovarian cancer pathogenesis remains unknown.

Materials and Methods: In this study, bioinformatic datasets were used to predict the expression of RHPN2 in clinical samples and determine the relationship between RHPN2 and the prognosis of ovarian cancer patients. Clinical samples were used to verify the prediction. RHPN2-targeting shRNA was used to investigate the effect of RHPN2 on ovarian cancer cells, and following RHPN2 knockdown, the proliferative and migratory capacities of ovarian cancer cells were tested. To determine the downstream signalling target of RHPN2, a luciferase reporter assay was conducted, and an animal experiment was carried out to confirm the effect of RHPN2 in vivo.

Results: The public datasets indicated that ovarian cancer tissues showed significantly higher RHPN2 expression than para-cancer normal tissues, and poor prognosis was observed in patients with higher RHPN2 expression, which was further confirmed in clinical samples. After RHPN2 was knocked down, the proliferation and migration of ovarian cancer cells were significantly impaired; a luciferase reporter assay indicated that the STAT3 signalling pathway was the most highly affected, and RHPN2 downregulation inhibited STAT3 nuclear translocation. STAT3 inhibitors partially rescued the tumourpromoting effect induced by RHPN2 overexpression, which was further confirmed by animal experiments.

Conclusion: Collectively, our results indicate that RHPN2 promotes malignant behaviours in ovarian cancer by activating STAT3 signalling.

Keywords: ovarian cancer, STAT3, RHPN2

\section{Introduction}

Globally, ovarian cancer is one of the most common cancers among females and the eighth leading cause of cancer-related deaths, with five-year survival rates below $45 \% .^{1-3}$ Ovarian cancer, which usually occurs in perimenopausal women, ranks third in incidence among all female cancers, second only to cervical and endometrial cancers. ${ }^{4}$ Approximately $60 \%$ of ovarian cancer patients are diagnosed in the late stage of disease, sometimes with abdominal metastasis. ${ }^{5}$ The risk factors for ovarian cancer include hormonal, reproductive and genetic predisposition and mutation in high penetrance genes. ${ }^{6,7}$ Developing early detection methods and identifying underlying driver genes are of great importance. 
STAT3, a member of the STAT family, is important for cell migration ${ }^{8}$ and is phosphorylated in different cell types by interleukin- 6 family proteins, epidermal growth factors, platelet-derived growth factors, hepatocyte growth factors, granulocyte colony-stimulating factors, and leptins. ${ }^{9,10}$ STAT3 can also induce the progression of various cancers. Moreover, STAT3 activation has been reported to promote to ovarian cancer aggression, cellular proliferation and resistance to chemotherapy medications, ${ }^{11-13}$ but upstream signals that promote STAT3 phosphorylation and subsequent nuclear translocation remain largely unknown.

RHPN2, whose gene is located on chromosome 19q12-13, is known as a RhoA-binding protein, ${ }^{14,15}$ but its biological function is far from clear; it has been reported that RHPN2 advances mesenchymal transformation of malignant glioma, ${ }^{16}$ but its specific role in ovarian cancer remains unknown.

In this study, the effect of RHPN2 expression on the prognosis of ovarian cancer was evaluated with bioinformatic datasets, and clinical samples were used to further confirm the evaluation. Then, shRNA was used to investigate the effect of RHPN2 on cancer behaviours, and phenotypic experiments were used to study the effect of RHPN2 downregulation on the proliferation and migration of cancer cells. In addition, a luciferase reporter assay was used to study the underlying signalling pathway affected by RHPN2 downregulation.

We found that RHPN2 was overexpressed in cancer tissues compared with normal tissues, and a worse prognosis was observed in RHPN2-expressing patients. After RHPN2 was knocked down, the proliferation and migration of ovarian cancer cells were significantly impaired. Moreover, a luciferase reporter assay indicated that the STAT3 signalling pathway was most strongly affected, and RHPN2 downregulation inhibited STAT3 nuclear translocation. Taken together, our data show that RHPN2 promotes ovarian cancer progression by activating the STAT3 signalling pathway in vitro and in vivo.

\section{Materials and Methods Cell Culture}

Three ovarian cancer cell lines (OVCAR4, HO8910 and SKOV3) and one normal ovarian epithelial cell line (IOSE80), procured from the Type Culture Collection China Centre, were used to evaluate theirRHPN2 expression, DMEM/F12 (Gibco, Carlsbad, USA) with 10\% FBS was used to culture all the cell lines. The cells were maintained in a $5 \% \mathrm{CO} 2$ incubator at a temperature of $37^{\circ} \mathrm{C}$.

\section{Transient Transfection}

Transient siRNA transfections and lentivirus infection were performed by Lipofectamine 2000 (Invitrogen, Carlsbad, USA). Isolation of the transfected cell clones was conducted after treatment with puromycin $(3 \mu \mathrm{g} / \mathrm{mL})$ for 2 weeks to establish cell lines. Lentiviral constructs expressing full-length RHPN2 were utilized in the overexpression experiments. The sequences of shRNAs used are as follows: Control, 5'- GCAAGCTGACCCTGAAG TT-3'; Sh-RHPN2-1, 5'- GCCGGAGTAAATTGCAGAA3'; Sh-RHPN2-2, 5'- CCTCTTGGCCTGAAGGAAA-3'.

\section{Quantitative Real-Time PCR (qRT-PCR)}

RNA extraction was performed using TRIzol (Takara, Japan). The cDNAs were synthesized by a reverse transcription kit (Invitrogen). qRT-PCR was conducted with SYBR Premix Ex Taq (Takara, Japan) and detected in an ABI 7500 Sequencing Detection System. GAPDH was used as a loading control. The $20-\mu \mathrm{L}$ PCR mixture consisted of $10 \mu \mathrm{L}$ of SYBR Premix Ex Taq (Takara, Japan), $2 \mu \mathrm{L}$ of cDNA, $0.4 \mu \mathrm{L}$ of $10 \mu \mathrm{M}$ sense and antisense primers, $0.4 \mu \mathrm{L}$ of ROX Reference Dye, and distilled water up to $20 \mu \mathrm{L}$. The optimized cycling program was 1 cycle of $95^{\circ} \mathrm{C}$ for $30 \mathrm{~s}, 40$ cycles of $95^{\circ} \mathrm{C}$ for $5 \mathrm{~s}$, and $60^{\circ} \mathrm{C}$ for $30 \mathrm{~s}$, and a final disassociation that was automatically added by 7500 System SDS software. The relative expression was calculated using the $2^{-\Delta \Delta \mathrm{CT}}$ method.

The following primer sequences were utilized: RHPN2, forward, 5'- AAGGGCTGTAATCCCCTTGC-3', reverse, 5'- CCGCACCTTTGAGTTTGTGG-3'; GAPDH forward, 5'- AATGGGCAGCCGTTAGGAAA-3', reverse, 5'- GCG CCCAATACGACCAAATC-3'; c-Myc, forward, 5'- ACTA ACATCCCACGCTCTGA-3', reverse, 5'- AAACCGCATC CTTGTCCTGT-3'; BIRC5, forward, 5'- TGAGAACGA GCCAGACTTGG-3', reverse, 5'- TTTCCTTTGCATGGG GTCGT-3'; VEGF, forward, 5'- ATGCGGATCAAACCT CACCA-3', reverse, 5'- CACCAACGTACACGCTCC AG-3'.

\section{Western Blot}

Western blotting was conducted as previously described, ${ }^{24}$ with GAPDH used as a loading control. The following primary antibodies were used: RHPN2 (H00085415-B01P, Novus, Cambridge, USA; 1:1000), GAPDH (ab181603, Abcam, Cambridge, USA; 1:1000), p-JAK2 (ab32101, Abcam; 1:1000), JAK2 (ab245303, Abcam; 1:1000), 
p-STAT3 (ab76315, Abcam; 1:1000) and STAT3 (ab119352, Abcam; 1:1000).

\section{Immunohistochemistry (IHC)}

Twenty normal ovarian samples (all obtained from postmenopausal women undergoing surgery for benign diseases) and twenty primary epithelial ovarian cancer samples (median age at primary diagnosis was 59 years (range 39-73)). Most patients presented with advanced disease at primary diagnosis [FIGO II: $\mathrm{n}=5$; FIGO III: $\mathrm{n}=15$ ]. Low and intermediate-grade samples $(\mathrm{n}=6$; highgrade $n=14$ ) that were formalin-fixed were obtained from the Affiliated Hospital of Qingdao University. IHC was conducted as previously described. ${ }^{24}$ The primary antibodies used were as follows: RHPN2 (NB600-792, Novus; 1:100) and p-STAT3 (ab76315, Abcam; 1:100).

\section{Immunofluorescence}

Briefly, OVCAR4 and HO8910 cells on glass coverslips were fixed with $4 \%$ paraformaldehyde, permeabilized with $0.4 \%$ Triton $\mathrm{X}-100$, and stained according to standard procedures. p-STAT3 (ab86430, Abcam; 1:500) and Alexa Fluor 488 (ab150113, Abcam; 1:800) were used for immunofluorescence. DAPI was used for nuclear staining. The cells were imaged by an FV-1000 laser-scanning confocal microscope (Olympus).

\section{Cell Proliferation Analysis}

Cells $\left(5 \times 10^{3} /\right.$ well $)$ were seeded in 96 -well plates. The CCK-8 reagent (Thermo Fisher Scientific) was used according to the manufacturer's protocol. Cells treated with CCK-8 reagent were cultured for 1.5 hours. The absorbance was measured at $450 \mathrm{~nm}$ with a microplate reader. The growth curves were determined by experiments in triplicate.

\section{Colony Formation Assays}

Cells $\left(1 \times 10^{3} /\right.$ well $)$ were seeded in 6 -well plates for 2 weeks and then stained with $0.4 \%$ crystal violet.

\section{Transwell Assays}

The lower chamber was filled with regular medium (750 $\mu \mathrm{L}$, with $10 \% \mathrm{FBS}$ ), and the upper chamber was seeded with a cell suspension in serum-free medium $(500 \mu \mathrm{L})$. After 24 hours of incubation, the cells that migrated onto the bottom layer were stained with $0.4 \%$ crystal violet. The migratory cells were quantified with five random fields under $10 \times$ magnification.

\section{Luciferase Reporter Assays}

Cells $\left(5 \times 10^{3} /\right.$ well $)$ were plated in $96-$ well plates. The Elk1-SRF, AP-1, NF-Kb, STAT3 and TCF/LEF luciferase reporter plasmids (Hanbio, Shanghai, China) and Renilla reporter vectors (Promega; Madison, USA) were cotransfected into the cells with Lipofectamine 3000 (Thermo Fisher Scientific). After 18 hours, the luciferase activity was detected by the Dual-Luciferase Assay Kit (Promega) and was normalized to Renilla activity.

\section{Mouse Xenograft Tumour Assay}

All the mouse experiments were approved by the Animal Care and Use Committee of Qingdao University. Fourweek-old BALB/c nude mice were obtained from Beijing Huafukang Bioscience, and 12 mice were randomly divided into 2 groups. We resuspended tumour cells in PBS, mixed the cells with Matrigel in a 1:1 ratio and injected the suspension into the subcutaneous right groin tissue of the BALB/c nude mice. After these mice developed a visible tumour mass, the tumour volumes were calculated as (length $\times$ width $\times$ width $) / 2$. Thirty days after subcutaneous injection, the mice were sacrificed.

\section{Statistical Analysis}

All experiments were performed in triplicate independently. Statistical analyses were performed using unpaired twotailed Student's $t$-test, and data are presented as mean \pm SEM. The survival rates were calculated by Kaplan-Meier curves, and Log-rank tests were used to examine the differences in survival rates between the two groups. GraphPad Prism version 7.00 software program (GraphPad; La Jolla, USA) was used to analyze data. $\mathrm{P}$ values $<0.05$ were considered statistically significant and were denoted by “*”; $\mathrm{P}$ values $<0.01$ were denoted by “**”; $\mathrm{P}$ values $<0.001$ were denoted by “***” and $\mathrm{P}>0.05$ was considered not significant and was denoted by "n.s.".

\section{Results}

\section{RHPN2 Expression is Upregulated in Both Human Ovarian Cancer Tissues and Ovarian Cell Lines}

To investigate the possible effect of RHPN2 on ovarian cancer, the GEPIA dataset was used. The ovarian cancer tissues expressed higher levels of RHPN2 than the paracancerous normal tissues, as shown in Figure 1A. As shown in Figure 1B, ovarian cancer patients with higher RHPN2 expression had a worse prognosis compared with 
A

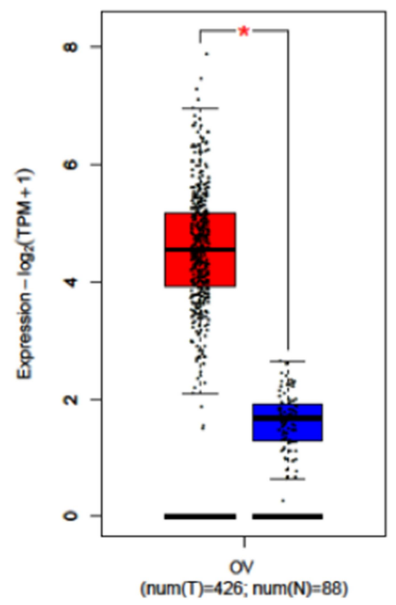

C

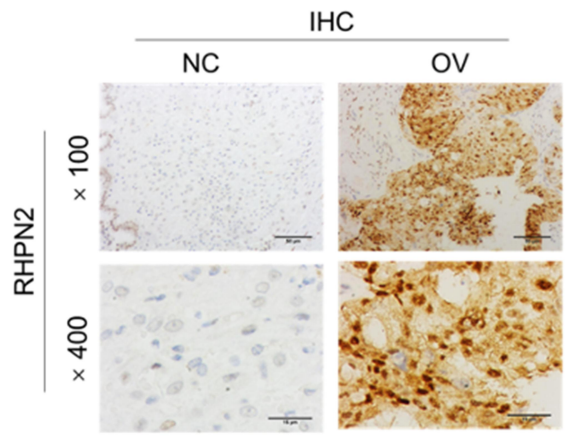

E

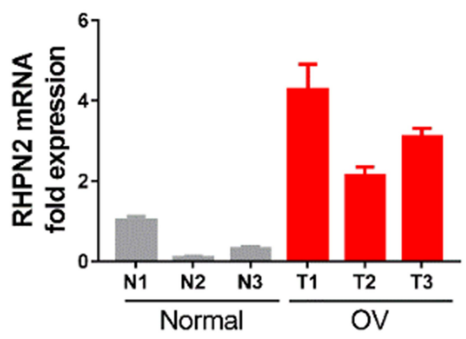

G

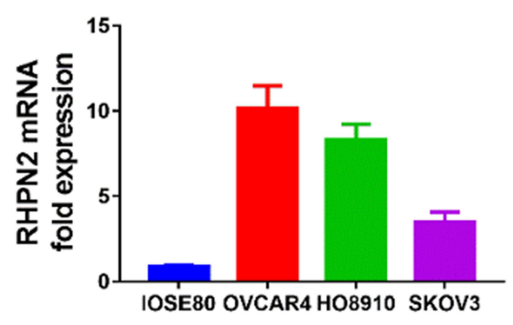

B
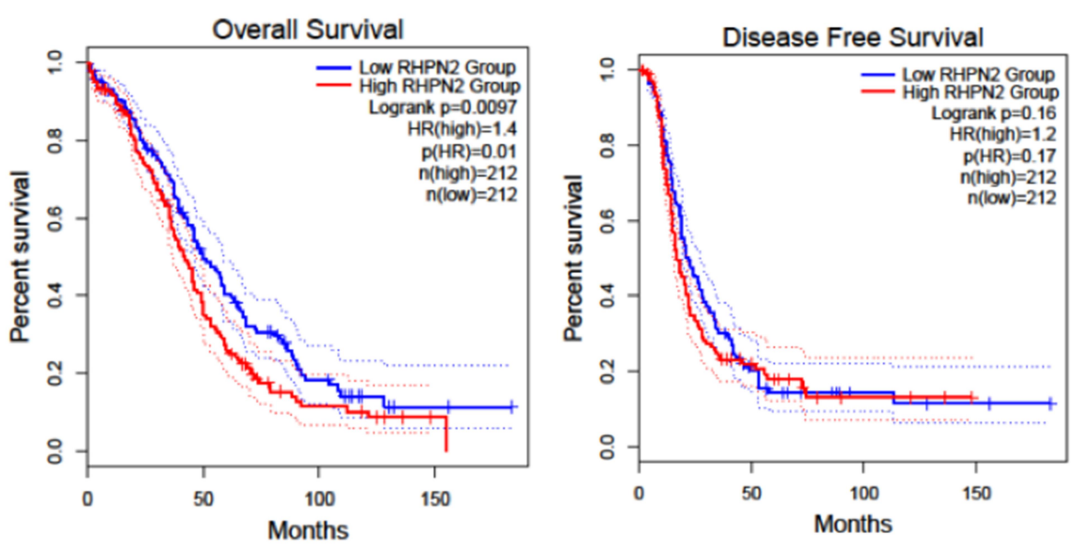

D

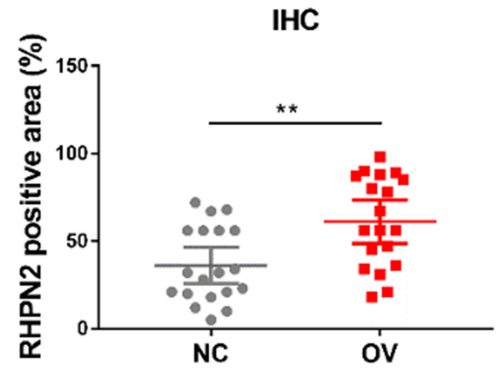

H
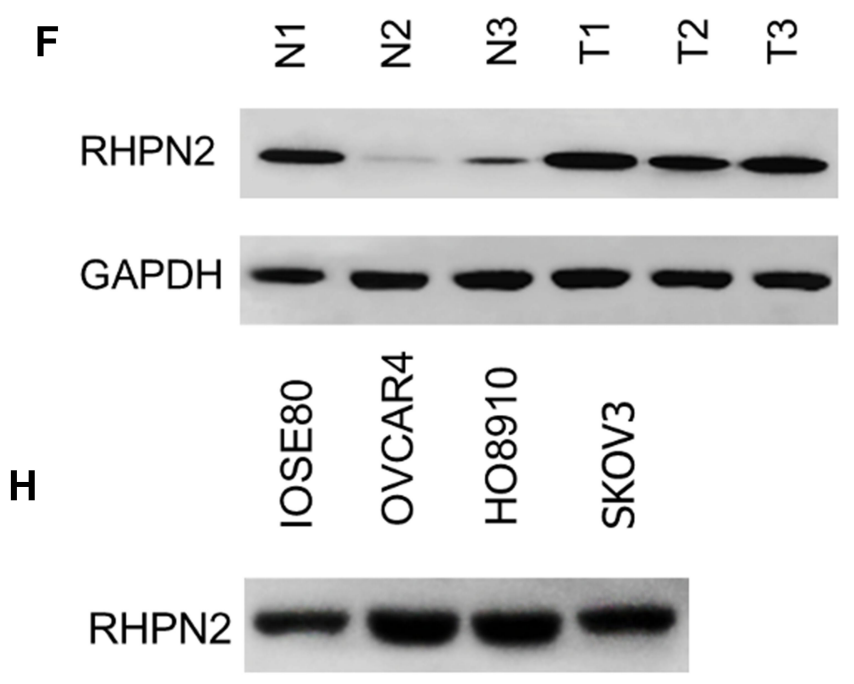

GAPDH

Figure I RHPN2 expression is upregulated in human ovarian cancer tissues and ovarian cell lines. (A) RHPN2 mRNA expression in ovarian cancer tissues ( $\mathrm{n}=426$ ) compared with that in normal tissues $(n=88)$. Data were from the GEPIA online database. (http://gepia2.cancer-pku.cn/). (B) Kaplan-Meier curves of overall survival (OS) and disease-free survival (DFS) obtained from GEPIA database. (C) Representative images of IHC staining of RHPN2 in normal ovarian tissues $(n=20)$ and ovarian cancer tissues (OV; $n=20)$. (D) Results of IHC analysis in ovarian tissues. (E) Relative RHPN2 mRNA expression of ovarian tissues from OV patients $(n=3)$ or normal controls $(n=3)$ as detected by qRT-PCR and $(\mathbf{F})$ Western blot. (G) Relative RHPN2 mRNA and $(\mathbf{H})$ protein expressions in cell lines (IOSE80, OVCAR4, HO8910 and SKOV3). * P<0.05, ** P<0.0I. 
patients with lower RHPN2 expression. To further confirm these results, clinical samples were used, as shown in Figure 1C. The immunohistochemical results indicated that compared with normal ovarian tissues, ovarian cancer tissues expressed higher RHPN2, which was further confirmed by Western blot and PCR analyses (Figure 1E, f); these results showed that RHPN2 was upregulated in ovarian cancer tissues both at the protein and mRNA levels.

As shown in Figure 1G-H, OVCAR4 and HO8910 had the highest protein and mRNA levels of RHPN2; therefore, OVCAR4 and HO8910 were selected for subsequent experiments.

\section{Silencing of RHPN2 Inhibits Malignant Cell Behaviours in Ovarian Cancer}

RHPN2-targeting shRNA was used to investigate the effect of RHPN2 expression in ovarian cancer cells. The results of RHPN2 knockdown are shown in Figure 2A and $\mathrm{B}$, indicating that RHPN2 was successfully silenced in all
A

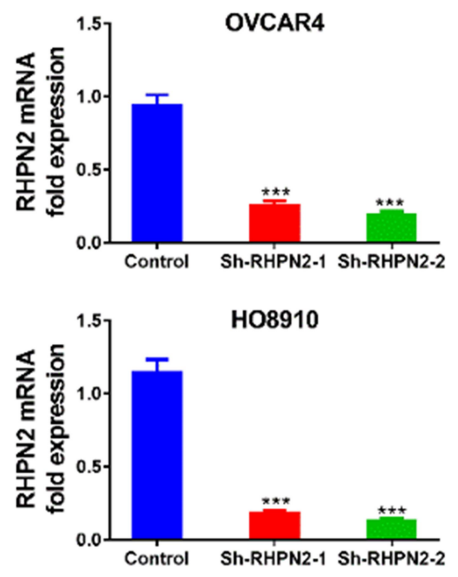

B

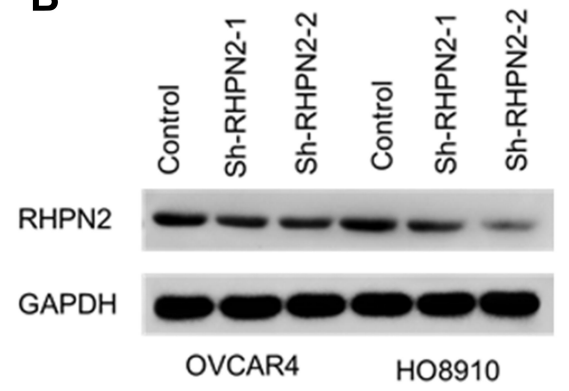

C
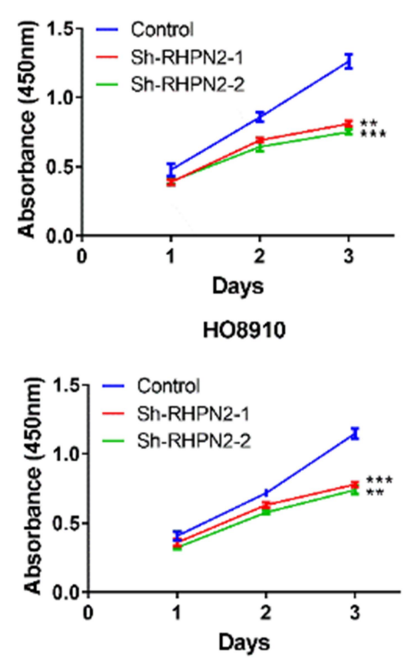

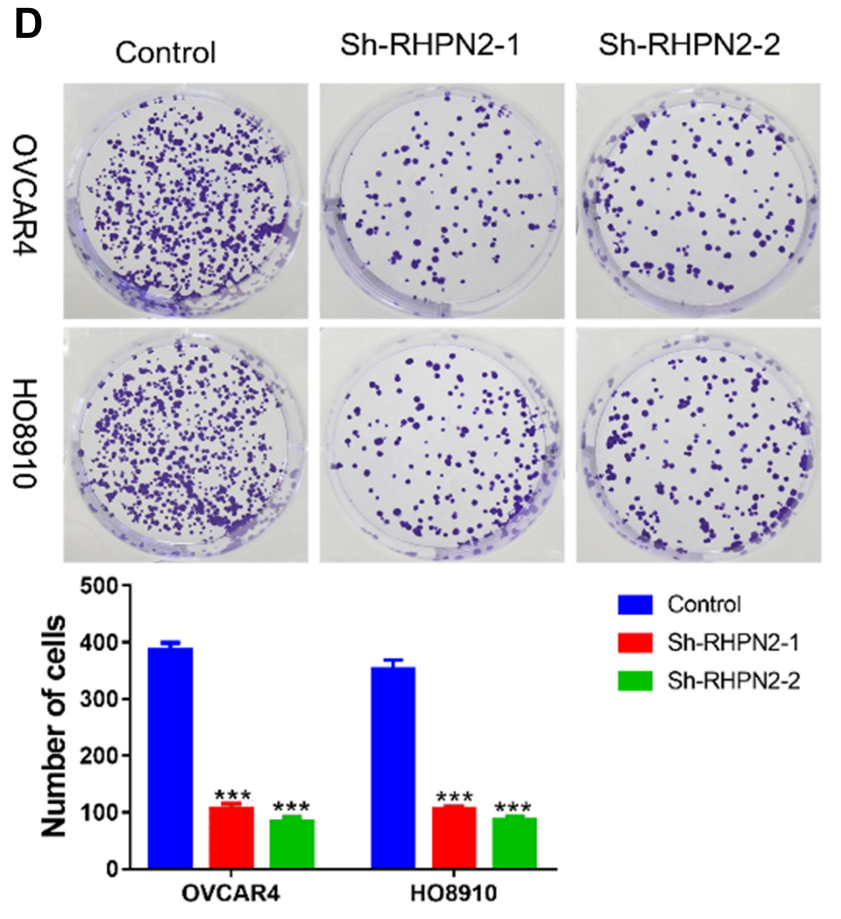

E

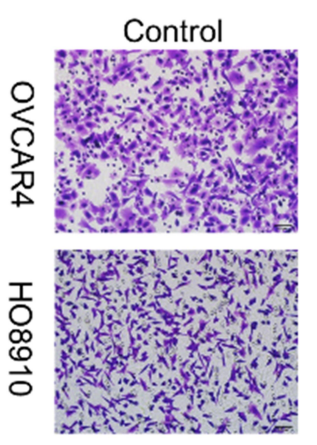

Sh-RHPN2-1

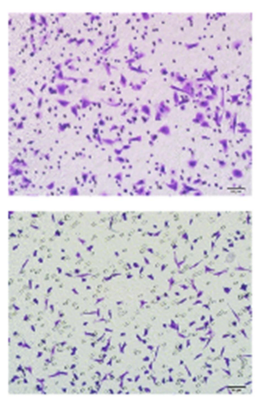

Sh-RHPN2-2
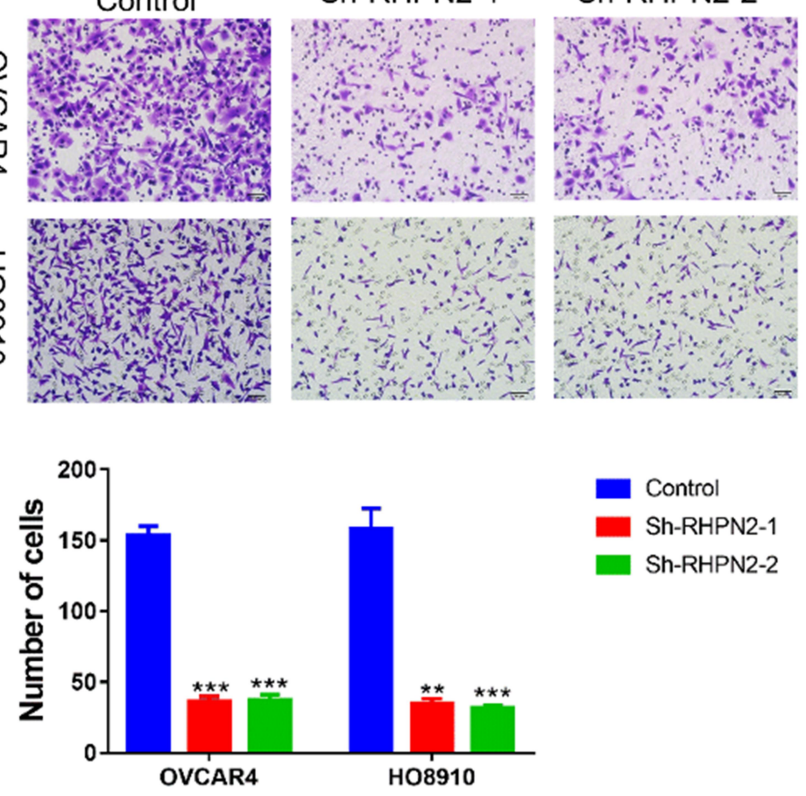

Figure 2 Loss of RHPN2 inhibits malignant cell behaviors in ovarian cancer. The results from qRT-PCR analysis (A) and Western blotting analysis (B) revealed RHPN2 expressions in OVCAR4 and HO89I0 cells transfected with lentiviruses containing two Short hairpin RNAs (Sh-RHPN2-I and Sh-RHPN2-2) or controls. (C) CCK-8 assays to evaluate cell proliferation. (D) Representative images and analysis of colony-forming assays in OVCAR4 and HO89I0 cells. (E) Transwell assays to evaluate cell migration and invasion. $* * \mathrm{P}<0.0 \mathrm{I}$, *** $\mathrm{P}<0.00 \mathrm{I}$. 
the cell lines. Colony formation and CCK-8 assays were used to evaluate the effect of RHPN2 expression on cellular proliferation. As shown in Figure 2C and D, downregulation of RHPN2 significantly inhibited the proliferation and colony formation of ovarian cancer cells, indicating that RHPN2 expression was necessary for ovarian cancer cell proliferation. To investigate the effect of RHPN2 expression on cellular migration, transwell experiments were performed. As shown in Figure 2E, downregulation of RHPN2 inhibited the migration of ovarian cancer cells in all the cell lines.

Taken together, our results showed that silencing RHPN2 inhibits malignant cell behaviours in ovarian cancer.

\section{RHPN2 Knockdown Reduces STAT3 Signalling by Suppressing STAT3 Nuclear Localization}

To determine the downstream signalling pathways of RHPN2, OVCAR4 cells with RHPN2 stably knocked down were transfected with Elk1, AP-1, NF-кB, STAT3 and TCF/LEF luciferase reporter plasmids. As shown in Figure 3A, the activation of the STAT3 luciferase reporter was more significantly decreased by RHPN2 downregulation than the activation of the other reporter plasmids, indicating that JAK2/STAT3 could serve as a downstream signalling pathway of RHPN2. To further confirm this finding, Western blotting was performed. As shown in Figure 3B, after downregulation of RHPN2, phosphorylation of JAK2 and STAT3 was significantly decreased; the classical downstream signalling of the JAK2/STAT3 pathway was evaluated (Figure 3C), showing that downregulation of RHPN2 also resulted in decreased transcriptional activity of JAK2/STAT3 downstream signalling. STAT3 is a transcription activator requiring nuclear translocation for its intended function. The immunofluorescence assay showed that nuclear translocation of STAT3 was inhibited when RHPN2 was downregulated (Figure 3D). Clinical samples were used to further confirm this conclusion. The association between STAT3 phosphorylation and RHPN2 expression was observed in ovarian cancer tissue, as shown in Figure 3E.

Taken together, our results confirm that RHPN2 depletion reduces STAT3 signalling through STAT3 nuclear localization.

\section{Re-Expression of RHPN2 Restores Malignant Cell Behaviours in Ovarian Cancer Cells, and STAT3 Inhibitor Reverses This Effect}

A specific JAK2/STAT3 inhibitor, AG490, was used to further confirm whether RHPN2 induced malignant cell behaviours in ovarian cancer by activating the STAT3 signalling pathway. A luciferase reporter assay showed that JAK2/STAT3 signalling was inhibited by RHPN2 downregulation (Figure 4A), as expected; transfection with the RHPN2-expressing vector rescued the activation of JAK2/STAT3, and administration of AG490 once again inhibited JAK2/STAT3 activation. Phenotypic experiments confirmed the effect of alterations in the signalling pathway on the malignant properties of ovarian cancer cells. As shown in Figure 4B and C, downregulation of RHPN2 inhibited the proliferation of ovarian cancer cells; after RHPN2 was re-expressed, the proliferation capacity of the cells was partly rescued, and after administration of the STAT3 inhibitor, the proliferation capacity of the cells was once again inhibited. As shown in Figure 4D, similar results were observed in the case of migratory capacity. RHPN2 knockdown impaired the migration of ovarian cancer cells; re-expression of RHPN2 rescued the migratory capacity of the cells, and this capacity was once again inhibited by administration of the STAT3 inhibitor.

Collectively, our results confirm that the reexpression of RHPN2 restores the malignant behaviours of ovarian cancer cells and that STAT3 inhibitors reverse this effect.

\section{RHPN2 Promotes Tumour Proliferation in vivo}

To further confirm the effect of RHPN2 expression in vivo, a xenograft model was constructed. OVCAR4 cells, shRHPN2-OVCAR4 cells and shRHPN2-OVCAR4 + RHPN2 cells were injected into the flanks of BALB/c $(\mathrm{nu} / \mathrm{nu})$ mice. Half of the mice injected with shRHPN2OVCAR4+RHPN2 were randomly selected and administered AG490 (STAT3 inhibitor) for 3 weeks. The mice were sacrificed 4 weeks later.

As shown in Figure $5 \mathrm{~A}-\mathrm{C}$, the proliferation rate and tumour weight were reduced when RHPN2 was downregulated; after RHPN2 was re-expressed, the tumour proliferation rate in vivo was rescued, and when AD490 was 
A

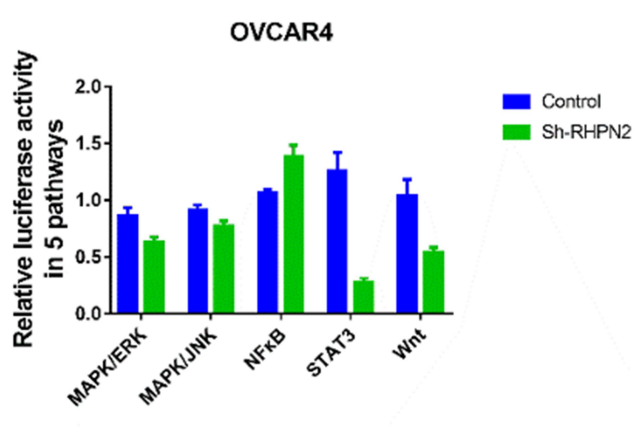

B

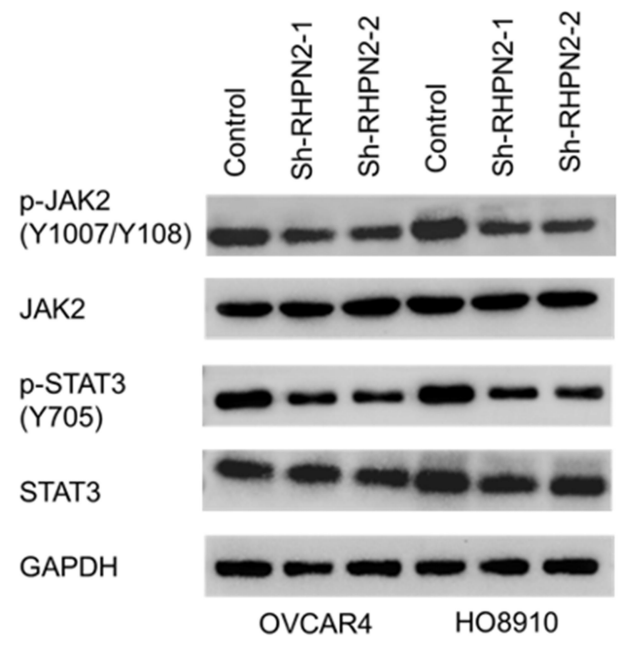

D

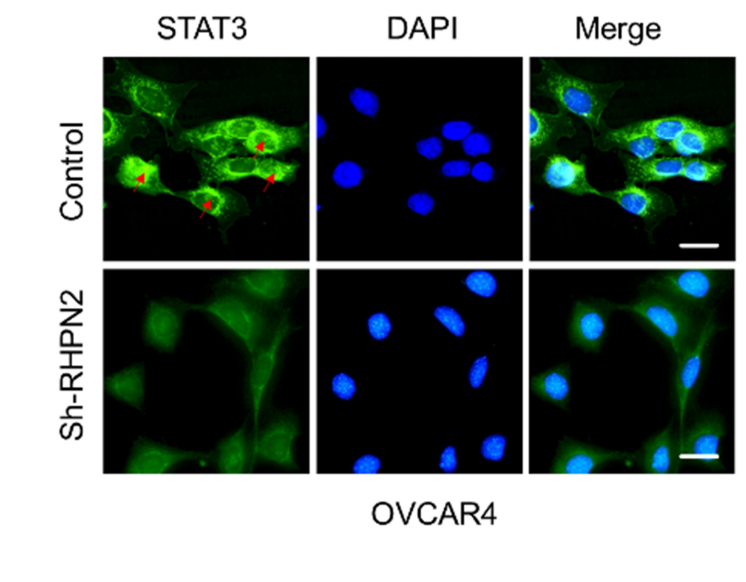

E

C

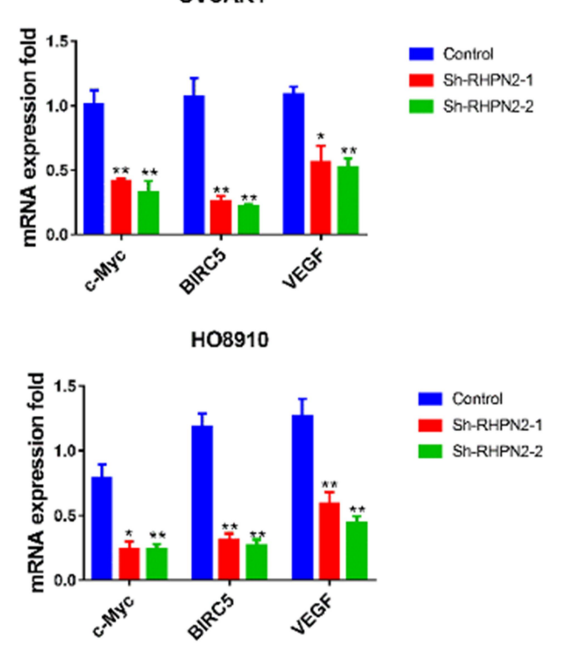

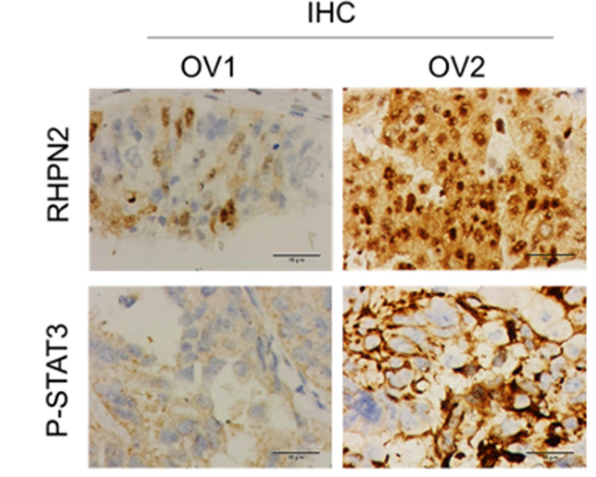

Figure 3 RHPN2 depletion reduces STAT3 signaling through suppressing STAT3 nuclear localization. (A) Relative luciferase activity in 5 pathways in OVCAR4 cells with RHPN2 knockdown. (B) Western blotting analysis of P-JAK2, JAK2, p-STAT3 and STAT3 in OVCAR4 and HO89I0 cells. GAPDH was used for normalization. (C) qRT-PCR analysis of expressions of STAT3 target genes (c-Myc, BIRC5, and VEGF). (D) Representative images from cell immunofluorescence for RHPN2. Nuclei stained with DAPI were blue. Red arrows in image highlight phosphorylation nuclear location STAT3. (E) Representative images of IHC staining of p-STAT3 and RHPN2 in paired ovarian cancer tissues (OV; $\mathrm{n}=20)$. $* \mathrm{P}<0.05$, ** $\mathrm{P}<0.01$.

administered, the tumour proliferation rate was once again inhibited. As shown in Figure 5D, immunohistochemical analysis further confirmed that Ki-67 expression was decreased when RHPN2 was downregulated, restored after RHPN2 was re-expression, and decreased again when AD490 was administered. 


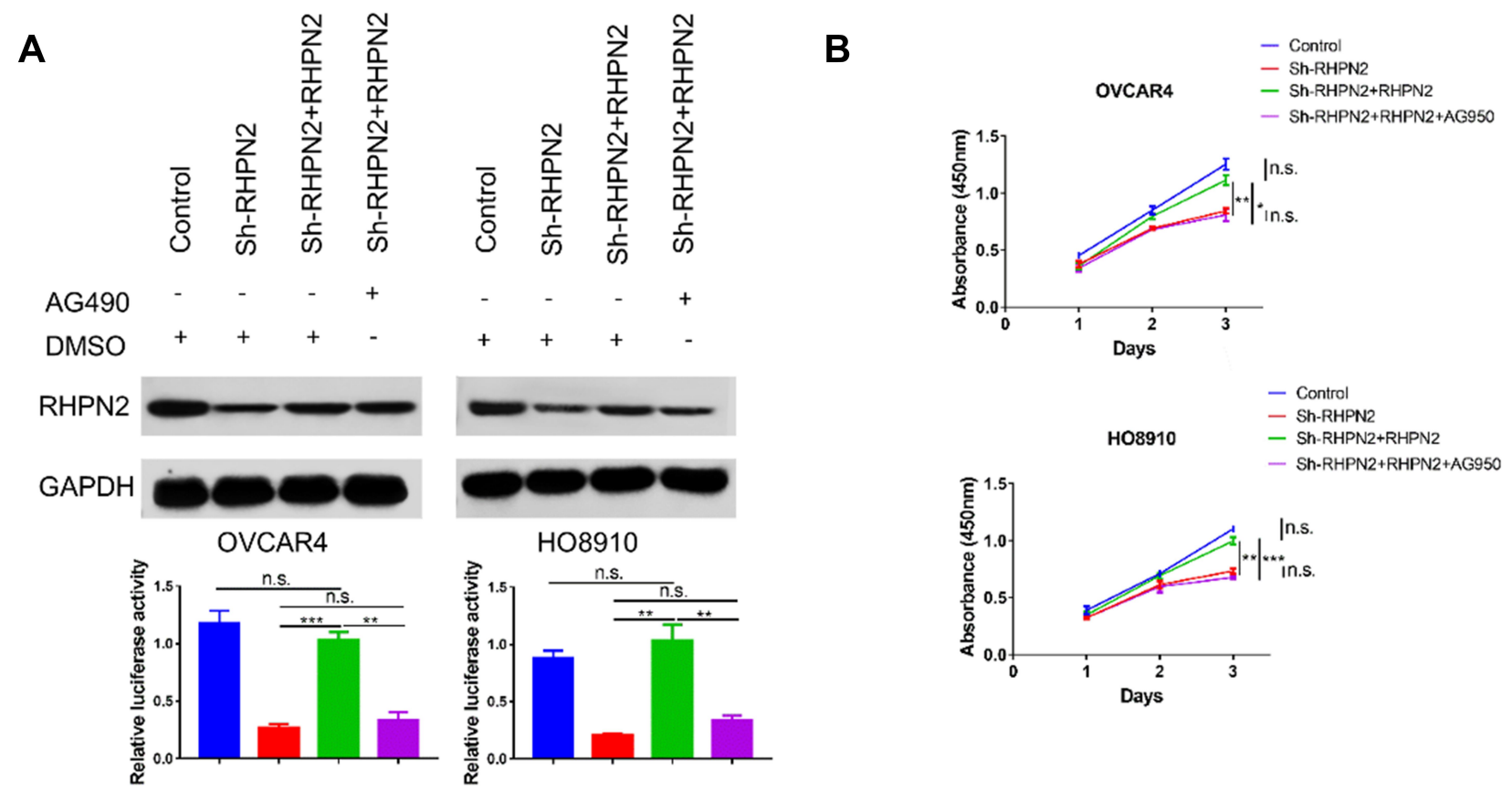

C

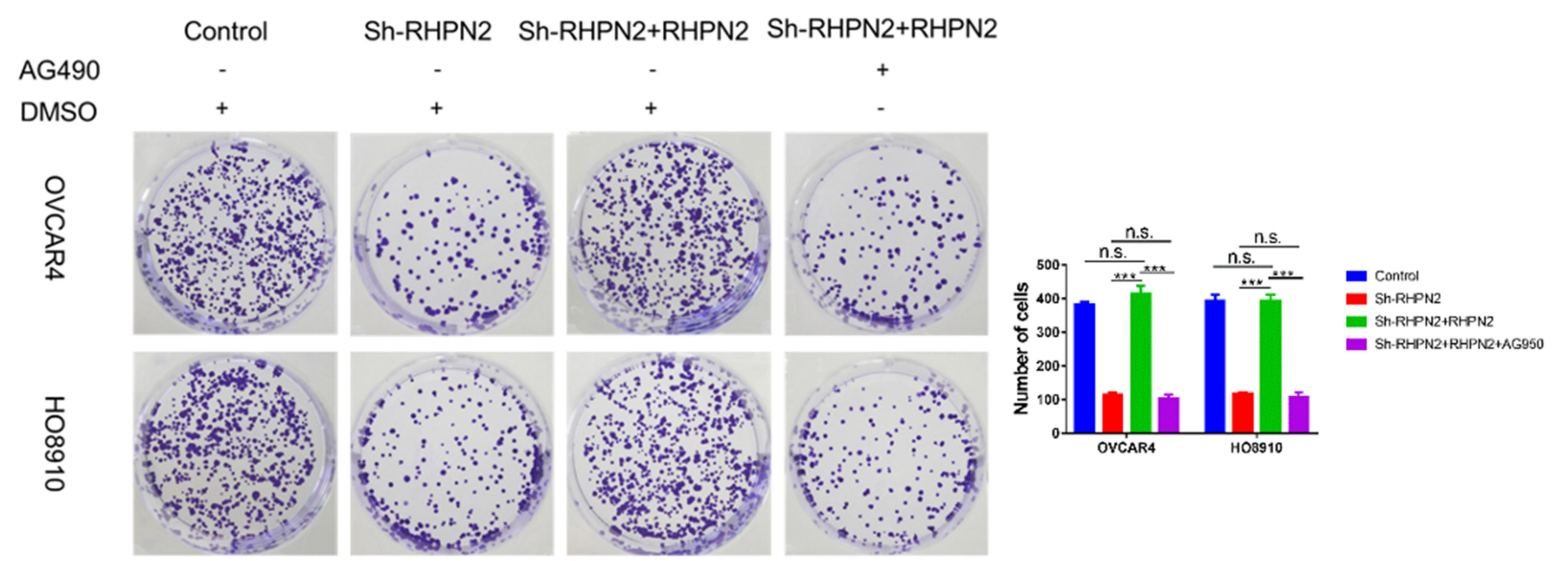

D

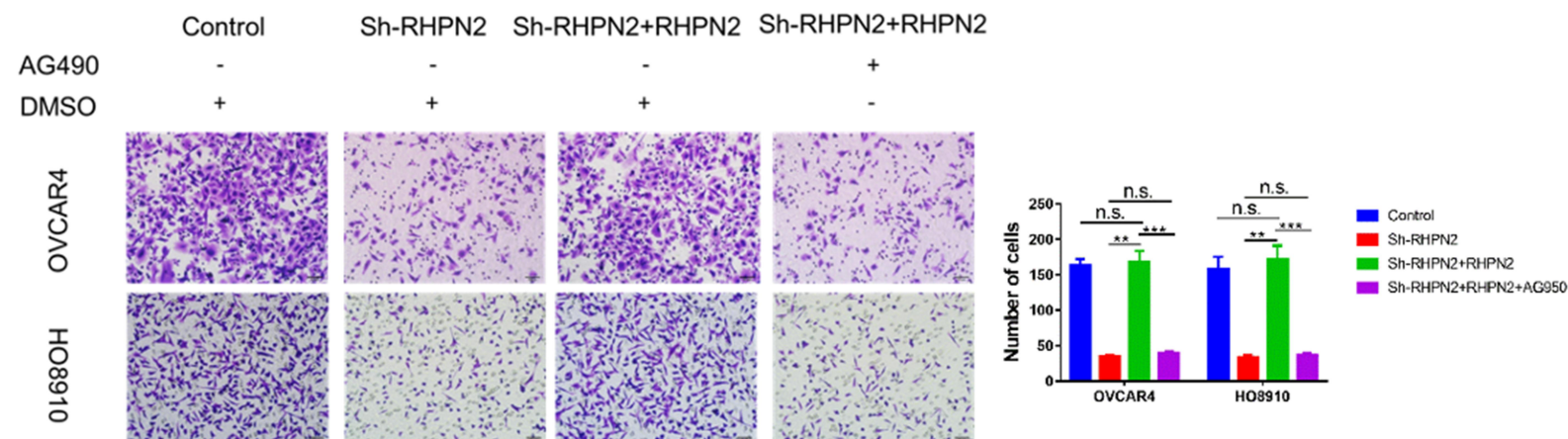

Figure 4 Re-expression of RHPN2 restores malignant properties in ovarian cancer cells and STAT3 inhibitor abolishes this effect. (A) The efficiency of stably transfected OVCAR4 and $\mathrm{HO} 8910$ cells with different treatments (AG490,40 $\mu \mathrm{mol} / \mathrm{L}$ or DMSO) were validated by Western blot and luciferase reporter assays. (B) The cell proliferation was evaluated by CCK-8 assays and (C) Representative images and analysis of colony-forming assays. (D) Representative images and results of transwell assays. ${ }^{*} \mathrm{P}<0.05$, ${ }^{* *} \mathrm{P}<0.01, * * * \mathrm{P}<0.00 \mathrm{I}$.

Taken together, our results confirm that RHPN2 promotes tumour proliferation in vivo by activating the JAK2/ STAT3 pathway.

\section{Discussion}

Ovarian cancer is the most lethal of all gynaecologic malignancies. ${ }^{17}$ Nearly 250,000 new cases and 152,000 
A

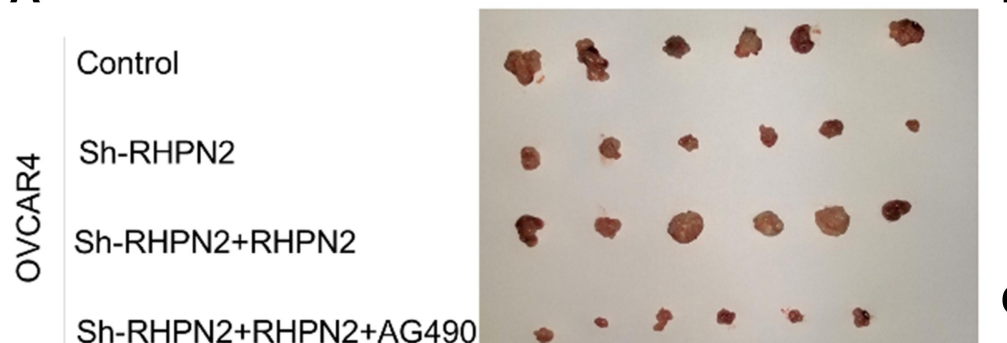

D

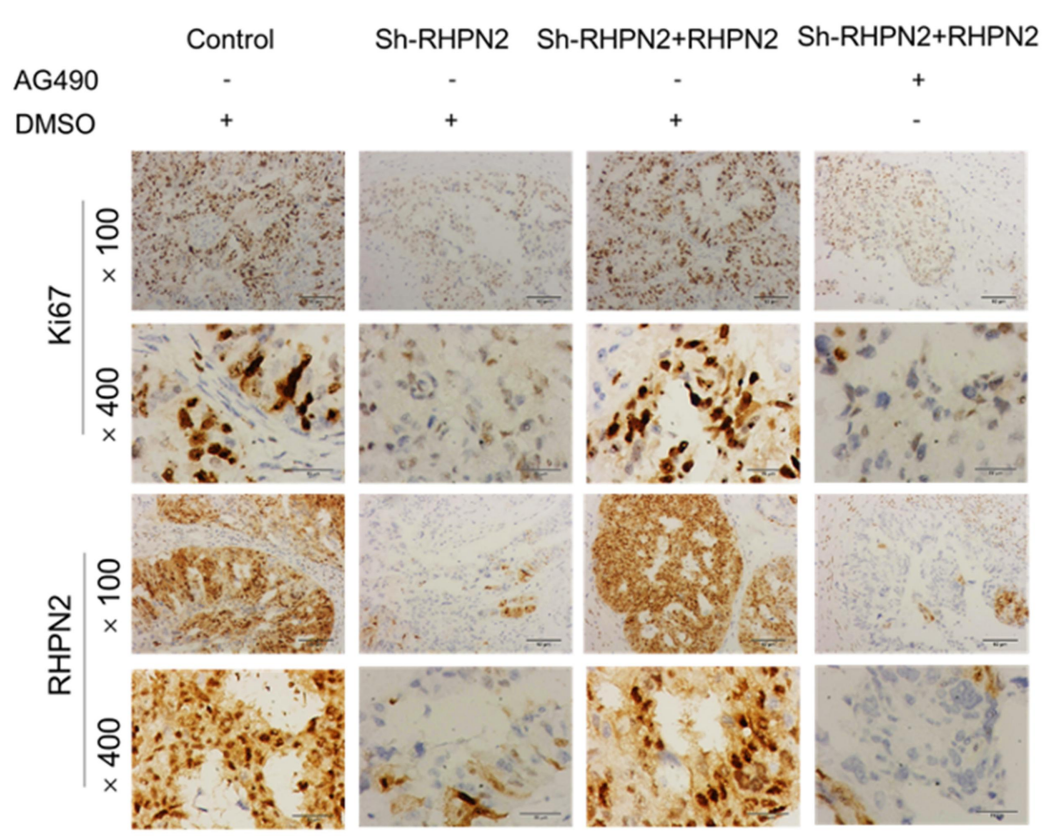

Sh-RHPN2+RHPN2+AG490

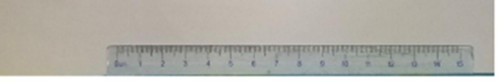

AG490
B

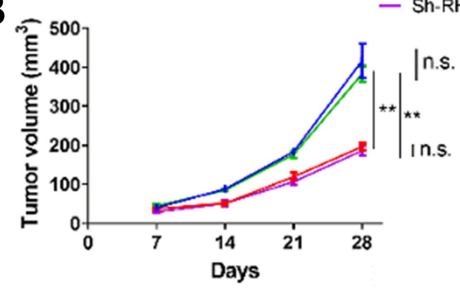

C

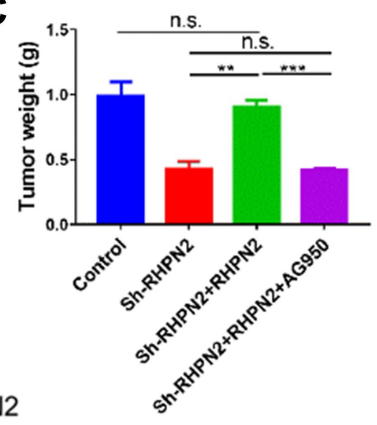

- Control

- Sh-RHPN2

- Sh-RHPN2+RHPN2

- Sh-RHPN2+RHPN2+AG950

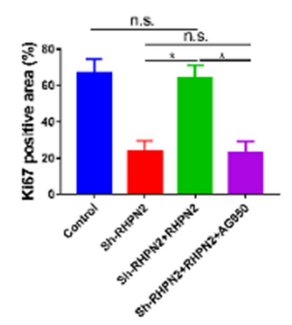

- Coritrol

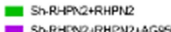

E SMREEND.RHPADiAGGSO

Figure 5 RHPN2 promotes tumor proliferation in vivo. (A) OVCAR4, OVCAR4-shRHPN2, OVCAR4-shRHPN2+RHPN2 overexpression were subcutaneously injected into BALB/c nude mice, thirty days after subcutaneous injection, mice were sacrificed. Representative tumour images at the end of the experiment are presented. (B) Tumor volume growth curves are presented. (C) Quantification of tumor weight is presented. (D) Representative IHC staining of Ki67 and RHPN2 in the four groups is presented. The right histogram presents percentage of stain positive area in the groups. The data are presented as means and SD. $* \mathrm{P}<0.05 ; * *, \mathrm{P}<0.0 \mathrm{I}, * * * \mathrm{P}<0.00 \mathrm{I}$.

deaths from ovarian cancer are observed around the world each year. Despite the rise in survival rates over the last four decades, $2 / 3$ of females still die less than 10 years after diagnosis. The 5-year survival rate is below $20 \%$ among females diagnosed with late-stage ${ }^{18-20}$ invasive epithelial ovarian cancer but surpasses $90 \%$ in those diagnosed at stage I. ${ }^{21,22}$ Therefore, developing early detection methods and identifying genes that promote ovarian cancer progression are of great importance.
RHPN2 is reported to be a RhoA-binding protein expressed by genes localized on chromosome 19q1213, but its biological function is largely unknown. Several reports indicated that RHPN2 expression alone was inadequate for carcinomatous change but could affect the malignant behaviours of cancer cells. However, to the best of our knowledge, no study has investigated the underlying role played by RHPN2 in ovarian cancer pathogenesis. 
Jiang et al reported that miR-205 suppresses the migration and proliferation of prostate cancer cells by downregulating RHPN $2,{ }^{15}$ indicating that RHPN2 may function as a tumour-promoting gene in prostate cancer. Similarly, in this study, we confirmed that RHPN2 is overexpressed in ovarian cancer tissues compared to normal tissues. After RHPN2 knockdown, decreased proliferation and migratory capacity can be observed in ovarian cancer cells, suggesting that RHPN2 promotes the progression of ovarian cancer, as in the case of prostate cancer.

Danussi et al reported that RHPN2 advances mesenchymal transition in malignant glioma by activating RhoA, which affects the organization of the actin cytoskeleton and subsequently promotes cancer cell invasion and migration. In this study, a similar role of RHPN2 was observed in ovarian cancer; RHPN2 knockdown significantly decreased the migratory and invasive capacities of ovarian cancer cells. In this case, JAK2/STAT3 was found to act as a downstream signalling target of RHPN2.

The STAT family includes seven members (STAT1-6 and STAT $5 \alpha / \beta$ ), all of which contain conserved domains, such as the N-terminal coiled-coil domain, DNA binding domain, linker, SH2 domain, and C-terminal transactivation domain. Of all the STAT members, STAT3 is ubiquitously expressed and regulates genes that affect the proliferation, survival, and differentiation of cells. Typically, STAT3 is activated through interactions between cell factors and their corresponding receptors. For instance, IL-6 family members can effectively activate STAT3, mediating its signal through the gp130 subunit, which trans-phosphorylates and activates JAK2, which can, in turn, activate STAT3 by phosphorylating its Tyr705 residue, inducing STAT3 to homodimerize, translocate to the nucleus, bind DNA and initiate transcription.

In this study, we reported, for the first time, that RHPN2 promotes JAK2 phosphorylation and STAT3 activation and that the downregulation of RHPN2 inhibits the phosphorylation of JAK2/STAT3. The immunofluorescence assay confirmed that the nuclear translocation of STAT3 was decreased, STAT3-mediated activation of transcription was impaired, and the classical transcription products of STAT3 were downregulated. Furthermore, RHPN2 promoted the migration and proliferation of ovarian cancer cells, but this effect could partially be blocked by the administration of AG490, which is a JAK2/STAT3 inhibitor, indicating that the tumour-promoting function of RHPN2 was partly dependent on JAK2/STAT3 signalling. No protein in the JAK2/STAT3 signalling pathway has been reported to interact with RHPN2; therefore, RHPN2 may affect the activation of JAK2/STAT3 signalling through some mechanism that needs further investigation.

Kobayashi et al reported that AG490 could suppress the progression of murine ovarian cancer, ${ }^{23}$ which was further confirmed by a study using animal models. Similar results were observed in this study: AG490 abolished the proliferation-promoting effect induced by RHPN2 overexpression in vivo. This result further confirmed our previous finding that RHPN2 promotes tumour progression in vivo, and this effect partly depends on STAT3 signalling.

Taken together, our results confirm that RHPN2 promotes malignant cell behaviours in ovarian cancer by activating STAT3 signalling and could serve as a possible therapeutic target for the treatment of ovarian cancer.

\section{Data Sharing Statement}

The datasets used and/or analyzed during the current study are available from the corresponding author Yankui Wang on reasonable request.

\section{Ethics Approval and Consent to Participate}

The study was approved by the Ethics Committee of the Qingdao University Affiliated Hospital and was carried out in compliance with the guidelines of the Helsinki Declaration of 2000. Animal experiments were approved by the Animal Care and Use Committee of Qingdao University and were performed in accordance with the Laboratory animal - Guideline for ethical review of animal welfare (GB/T 35892-2018).

\section{Acknowledgments}

We thank the Department of Gynecology, the Affiliated Hospital of Qingdao University, Qingdao, China, for providing the tissue samples.

\section{Author Contributions}

All authors made substantial contributions to conception and design, acquisition of data, or analysis and interpretation of data; took part in drafting the article or revising it critically for important intellectual content; agreed to submit to the current journal; gave final approval of the version to be published; and agree to be accountable for all aspects of the work. 


\section{Funding}

Research reported in the article received no funding.

\section{Disclosure}

The authors report no conflicts of interest in this work.

\section{References}

1. Kossaï M, Leary A, Scoazec JY, Genestie C. Ovarian Cancer: A Heterogeneous Disease. Pathobiology. 2018;85(1-2):41-49. doi:10. $1159 / 000479006$

2. Menon U, Karpinskyj C, Gentry-Maharaj A. Ovarian Cancer Prevention and Screening. Obstet Gynecol. 2018;131(5):909-927. doi:10.1097/AOG.0000000000002580

3. Rooth C. Ovarian cancer: risk factors, treatment and management. $\mathrm{Br}$ J Nurs. 2013;22:S23-S30.

4. Eisenhauer EA. Real-world evidence in the treatment of ovarian cancer. Ann Oncol. 2017;28:viii61-viii65. doi:10.1093/annonc/mdx 443

5. Ottevanger PB. Ovarian cancer stem cells more questions than answers. Semin Cancer Biol. 2017;44:67-71. doi:10.1016/j.semcancer. 2017.04.009

6. Stewart C, Ralyea C, Lockwood S. Ovarian Cancer: an Integrated Review. Semin Oncol Nurs. 2019;35(2):151-156. doi:10.1016/j. soncn.2019.02.001

7. Webb PM, Jordan SJ. Epidemiology of epithelial ovarian cancer. Best Pract Res Clin Obstet Gynaecol. 2017;41:3-14. doi:10.1016/j.bpobgyn. 2016.08.006

8. Yu H, Pardoll D, Jove R. STATs in cancer inflammation and immunity: a leading role for STAT3. Nat Rev Cancer. 2009;9(11):798-809. doi: $10.1038 / \mathrm{nrc} 2734$

9. Yu H, Lee H, Herrmann A, Buettner R, Jove R. Revisiting STAT3 signalling in cancer: new and unexpected biological functions. Nat Rev Cancer. 2014;14(11):736-746. doi:10.1038/nrc3818

10. Srivastava J, DiGiovanni J. Non-canonical Stat3 signaling in cancer. Mol Carcinog. 2016;55(12):1889-1898. doi:10.1002/mc.22438

11. Galoczova M, Coates P, Vojtesek B. STAT3, stem cells, cancer stem cells and p63. Cell Mol Biol Lett. 2018;23(1):12. doi:10.1186/s11658018-0078-0
12. Fan Y, Mao R, Yang J. NF-кB and STAT3 signaling pathways collaboratively link inflammation to cancer. Protein Cell. 2013;4 (3):176-185. doi:10.1007/s13238-013-2084-3

13. Fathi N, Rashidi G, Khodadadi A, Shahi S, Sharifi S. STAT3 and apoptosis challenges in cancer. Int J Biol Macromol. 2018; 117:993-1001. doi:10.1016/j.ijbiomac.2018.05.121

14. Chen C, Guo Q, Shi J, et al. Genetic variants of MGMT, RHPN2, and FAM49A contributed to susceptibility of nonsyndromic orofacial clefts in a Chinese population. J Oral Pathol Med. 2018;47 (8):796-801. doi:10.1111/jop.12758

15. Jiang S, Mo C, Guo S, et al. Human bone marrow mesenchymal stem cells-derived microRNA-205-containing exosomes impede the progression of prostate cancer through suppression of RHPN2. J Exp Clin Cancer Res. 2019;38(1):495. doi:10.1186/s13046-019-1488-1

16. Danussi C, Akavia UD, Niola F, et al. RHPN2 Drives Mesenchymal Transformation in Malignant Glioma by Triggering RhoA Activation. Cancer Res. 2013;73(16):5140. doi:10.1158/0008-5472.CAN-13-11 68-T

17. Adams SF, Benencia F. Immunotherapy for ovarian cancer: what are the targets of the future? Future Oncol. 2015;11(9):1293-1296. doi: $10.2217 /$ fon. 15.44

18. Hennessy BT, Coleman RL, Markman M. Ovarian cancer. Lancet. 2009;374(9698):1371-1382. doi:10.1016/S0140-6736(09)61338-6

19. Johnson N, Liao JB. Novel Therapeutics for Ovarian Cancer: the 11th Biennial Rivkin Center Ovarian Cancer Research Symposium. Int J Gynecol Cancer. 2017;27:S14. doi:10.1097/IGC.0000000000 001115

20. Karam AK, Karlan BY. Ovarian cancer: the duplicity of CA125 measurement. Nat Rev Clin Oncol. 2010;7(6):335-339. doi:10.1038/ nrclinonc. 2010.44

21. Matulonis UA. Ovarian Cancer. Hematol Oncol Clin North Am. 2018;32(6):xiii-xiv. doi:10.1016/j.hoc.2018.09.006

22. Wenham RM. Ovarian Cancer: A Bright Future. Cancer Control. 2011;18(1):4-5. doi:10.1177/107327481101800101

23. Kobayashi A, Tanizaki Y, Kimura A, et al. AG490, a Jak2 inhibitor, suppressed the progression of murine ovarian cancer. Eur J Pharmacol. 2015;766:63-75. doi:10.1016/j.ejphar.2015.09.039

24. Tang H, Chu Y, Huang Z, Cai J, Wang Z. The metastatic phenotype shift toward myofibroblast of adipose-derived mesenchymal stem cells promotes ovarian cancer progression. Carcinogenesis. 2019;41 (2):182-193. doi:10.1093/carcin/bgz083
OncoTargets and Therapy

\section{Publish your work in this journal}

OncoTargets and Therapy is an international, peer-reviewed, open access journal focusing on the pathological basis of all cancers, potential targets for therapy and treatment protocols employed to improve the management of cancer patients. The journal also focuses on the impact of management programs and new therapeutic agents and protocols on patient perspectives such as quality of life, adherence and satisfaction. The manuscript management system is completely online and includes a very quick and fair peer-review system, which is all easy to use. Visit http://www.dovepress.com/ testimonials.php to read real quotes from published authors. 